PALEO

Revue d'archéologie préhistorique

$20 \mid 2008$

Spécial table ronde ( $2^{\mathrm{e}}$ partie) : Le Gravettien : entités régionales d'une paléoculture européenne, Les Eyzies, juillet 2004

\title{
Le statut de la fléchette au sein des premières industries gravettiennes
}

Considering the flechette status within the first gravettian industries

\section{Damien Pesesse}

\section{OpenEdition}

Journals

Édition électronique

URL : http://journals.openedition.org/paleo/1572

DOI : $10.4000 /$ paleo. 1572

ISSN : $2101-0420$

Éditeur

SAMRA

Édition imprimée

Date de publication : 1 décembre 2008

Pagination : $277-290$

ISSN : $1145-3370$

Référence électronique

Damien Pesesse, «Le statut de la fléchette au sein des premières industries gravettiennes », PALEO

[En ligne], 20 | 2008, mis en ligne le 11 mai 2011, consulté le 07 juillet 2020. URL : http://

journals.openedition.org/paleo/1572 ; DOI : https://doi.org/10.4000/paleo.1572

\section{(c) (i) $\odot$}

PALEO est mis à disposition selon les termes de la licence Creative Commons Attribution - Pas d'Utilisation Commerciale - Pas de Modification 4.0 International. 


\title{
LE STATUT DE LA FLÉCHETTE AU SEIN DES PREMIĖRES INDUSTRIES GRAVETTIENNES
}

\author{
Damien PESESSE ${ }^{(1)}$
}

\begin{abstract}
Résumé : Les premières phases du Gravettien en France soulèvent de nombreuses interrogations liées au polymorphisme des industries attribuées à cette période. Ces gisements se distinguent notamment par la présence de fléchettes que l'on retrouve dans des proportions variables. Cet outil est fréquemment associé à d'autres fossiles directeurs du Gravettien, notamment des pointes de La Font-Robert et des burins de Noailles. Toutefois, ces associations relèvent fréquemment de contextes mal définis stratigraphiquement car la majorité des fouilles des gisements du Gravettien ancien se déroulèrent entre les années 1900 et 1940. Parmi les ensembles archéologiques homogènes figurent le gisement éponyme de La Gravette, l'Abri Pataud, Puy Jarrige et La Vigne Brun. À La Gravette, F. Lacorre reconnût un niveau dont la fléchette est la composante principale à l'exclusion de tout autre type d'armature. L'originalité de cette industrie et l'absence de points de comparaisons, y compris dans les niveaux supérieurs de La Gravette, le conduisît à l'individualiser sous le terme de Bayacien (Lacorre 1960). Une nouvelle étude de ce gisement permet de reconnaître l'intégrité de ce niveau, remise en question par de nombreux auteurs (Sonneville-Bordes 1958 ; Delporte 1972 ; Rigaud 1988). D'un point de vue technologique, la production de fléchettes s'avère prédominante, réalisée de manière autonome selon un schéma opératoire très spécifique. La production laminaire destinée à alimenter l'outillage de fonds commun se déroule selon d'autres logiques techniques, indépendantes. La situation diffère pour le gisement de La Vigne Brun. Les fléchettes se trouvent associées à des pointes de La Gravette et la production de ces armatures s'effectue dans un continuum technique, associant également la production de supports à finalités domestiques.

Ces données techniques permettent d'envisager de manière originale les premières phases du Gravettien. Elles révèlent que le Bayacien constitue effectivement une entité à part entière et légitime ainsi son individualisation au sein de la sphère gravettienne. Ce terme doit être compris dans cette acception réduite qui ne concerne, à l'heure actuelle, que le gisement doublement éponyme. Reconnaître l'originalité du Bayacien soulève de nombreuses implications d'ordre paléohistorique et modifie notre compréhension du passage de l'Aurignacien au Gravettien. Les restructurations importantes des systèmes techniques entre l'Aurignacien et le Gravettien peuvent être perçues comme le reflet des profondes mutations affectant ces sociétés du Paléolithique supérieur.
\end{abstract}

Mots-clés : Gravettien ancien, Bayacien, fléchette, technologie lithique.

Abstract: Flechette status within the first gravettian industries. The begining of the Gravettian rises many questions after the polymorphism of the industries attributed to this period. Early Gravettian sites are mainly distinguished by the presence of fléchettes, which are found in various proportions. Fléchettes are frequently associated to other « fossiles directeurs ", such as pointes de La Font-Robert and burins de Noailles. But the association of these tools occurs mainly in sites where stratrigraphical data are incomplete. Indeed, most of them has been excavated from 1900 to 1940, when stratrigraphic assessment was on its starts. La Gravette, L'Abri Pataud, Puy Jarrige and La Vigne Brun can be considered as homogenous archeological industries of the early Gravettian. In La Gravette, Fernand Lacorre recognized a level exclusively containing fléchettes, any other type of armature being absent. The originality of this layer lead Lacorre to name it Bayacien (Lacorre 1960). The integrity of this layer has been previously questioned by many authors (Sonneville-Bordes 1958 ; Delporte 1972 ; Rigaud 1988). In the present work, a reexamination of the lithic industry is reported, confirming its technological integrity. The production of fléchettes is predominant in this level and it is performed according to a very specific and normalized " schéma opératoire ". The laminar production, commited to compose the domestical tool kit, follows a different and independent scheme. The situation is different when the site of La Vigne Brun is considered. Here, the fléchettes are associated to the pointes de La Gravette, and the both are the result of a single « schéma opératoire " integrating also domestical tool kit in the same process.

These technological data allow to reconsider the early Gravettian. In fact, the Bayacien constitute a clear-cut unit, technically individualized into the Gravettian. At present, the Bayacien could be understood in a very restrictive acceptation, i.e. concerning exclusively the specific level of La Gravette. The recognition of the Bayacien rise many paleo-historical questions about the transition from Aurignacian to Gravettian. To exactly distinguish the Bayacien entails a modification of the way the transition from Aurignacian to Gravettian is represented. The deep modifications of the technical systems employed during the late Aurignacian, the Bayacien and the early Gravettian may reflect the complex social mutations occuring in that time. (Translation : Alfredo J. Zamora)

Key-words : Early Gravettian, Bayacien, fléchette, lithic technology.

(1) Maison méditerranéenne des Sciences de l'Homme LAMPEA - UMR 6636, 5 rue du Château de l'Horloge, BP 647, 13094 Aix-en-Provence Cedex 2 - dam_pes @yahoo.fr 


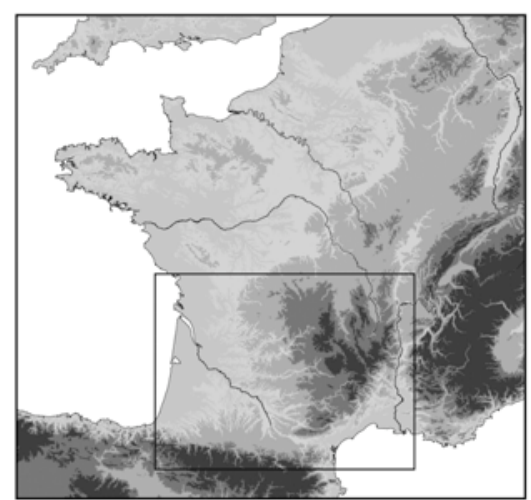

1 - Vigne Brun

6 - Puy Jarrige

11 - Vignaud

2 - Crouzade

7 - Font-Robert

12 - Poisson

3 - Bize

8 - Laussel

13 - Gravette

4 - Roc de

9 - Roque

14 - Durand-Ruel

Gavaudun

Saint-Christophe

15 - Vachons

5 - Noailles

10 - Pataud

16 - Pair-non-Pair

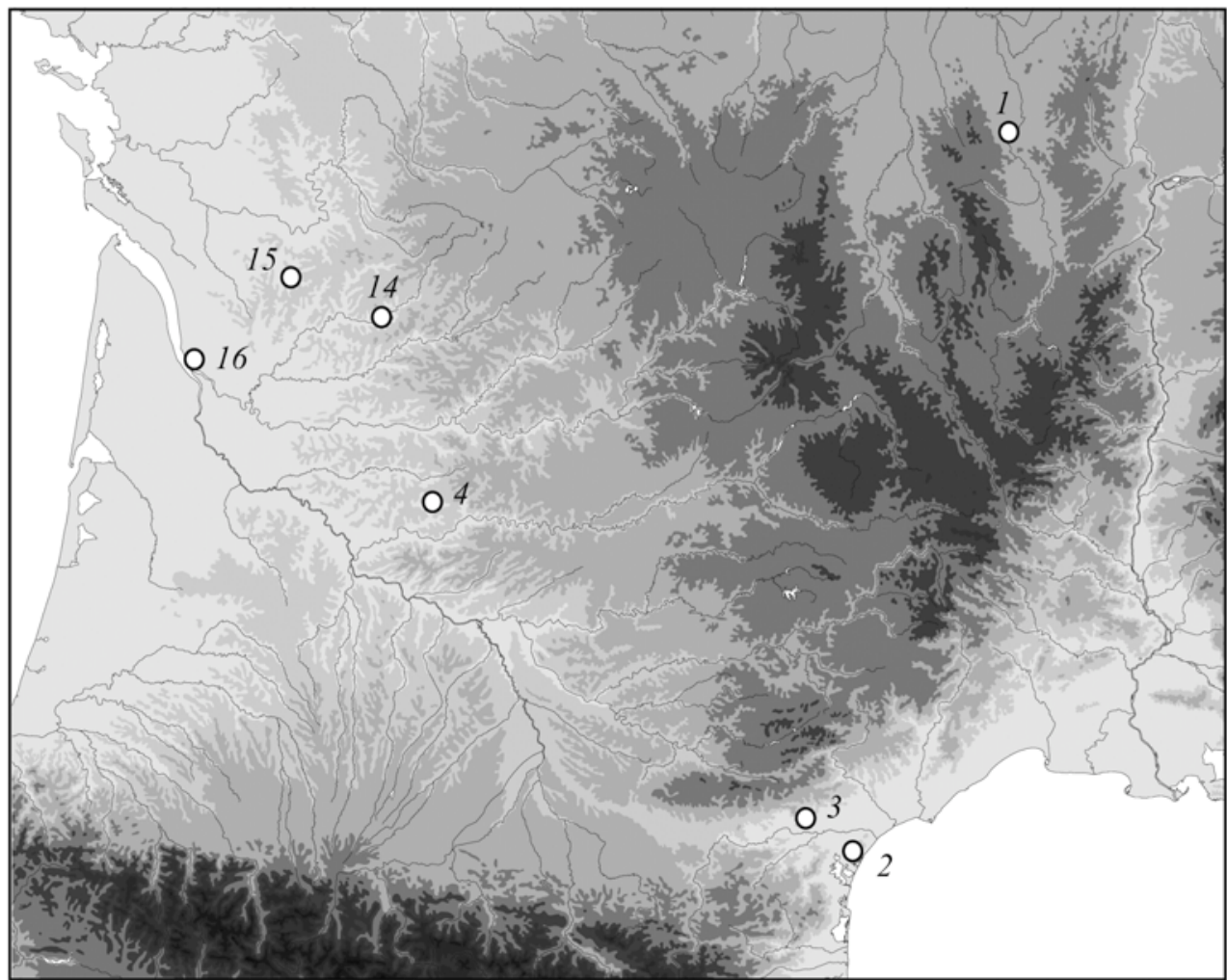

50

Echelles en km

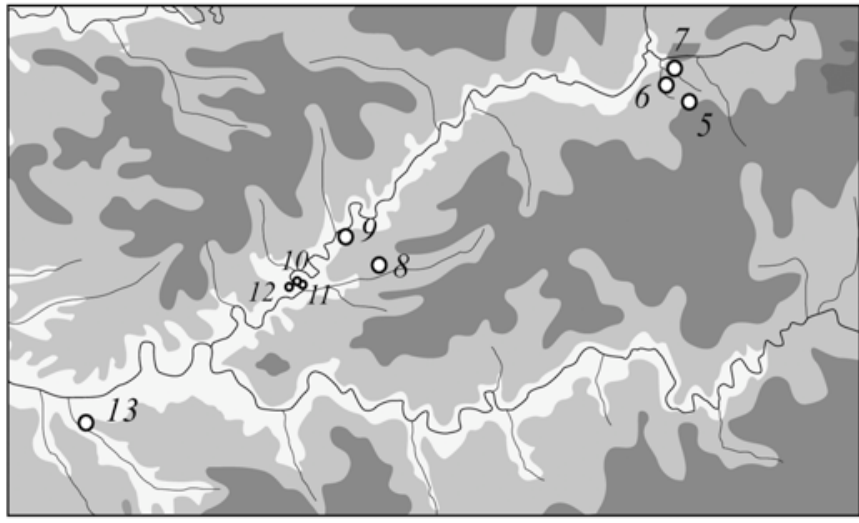

Figure 1 - Répartition des gisements à fléchettes.

Figure 1 - Geographic distribution of fléchette sites. 


\section{INTRODUCTION}

La stratigraphie de La Gravette occupe une place princeps dans la compréhension des tous premiers stades du Gravettien. Fernand Lacorre reconnût avec une certaine clairvoyance l'importance du niveau à fléchettes (Lacorre 1933a) situé entre un niveau d'Aurignacien évolué à burins busqués et trois niveaux de Gravettien à pointes de La Gravette principalement (Lacorre 1960). Cette observation fut confirmée lors de la fouille de l'Abri Pataud par Hallan M. Movius, vérifiant vingt ans plus tard la position des fléchettes à la base de la séquence gravettienne (Bricker 1995).

L'originalité de cette industrie amena F. Lacorre à l'individualiser sous le terme de «Bayacien ». Pour lui, ce niveau à fléchettes procède d'une lignée distincte du « Gravétien » mais parallèle (Lacorre 1959) puisque les données typologiques ne permettaient pas d'établir un lien direct entre ces entités. Le terme de "Bayacien " eut un avenir contrasté, retenu par certains auteurs compte tenu de l'originalité de cet assemblage (Delporte 1972 ; Otte 1981 ; Djindjian et Bosselin 1994 ; Bosselin 1996) ou refusé au profit de «Périgordien moyen » (Bricker 1974, 1995 ; Perpère 1992) car la stratigraphie de l'Abri Pataud ne présente pas une telle rupture entre niveaux à fléchettes et niveaux à pointes de La Gravette. L'appellation “Bayacien " fut également rejetée, compte tenu du manque d'éléments de caractérisation (Rigaud 1988). Aujourd'hui, la nature du Bayacien et donc son existence demandent encore à être définies.

L'étude du gisement éponyme constitue l'occasion d'aborder la structuration technique de cet assemblage bayacien. Cette attribution même s'avère problématique car les marges du Bayacien sont aussi floues que sa nature propre. Qu'est-ce qu'une industrie bayacienne? Existet-il d'autres gisements bayaciens ? Sur quelles bases et dans quelles limites, pouvons-nous parler de Bayacien ? Est-ce la présence ou une proportion de fléchettes suffisante qui définit le Bayacien? Comment s'articule l'évolution vers le Gravettien à pointes de La Gravette ? Les fléchettes sont-elles associées à d'autres " fossiles directeurs "? Ces interrogations sont inhérentes à la nature même du Gravettien qui, loin de constituer une entité, apparaît comme une « mosaïque » (Klaric 2003) de techno-complexes à connotations géographiques et chronologiques dont les liens sont encore à définir.

\section{État de la question}

Comme la majorité des sites gravettiens, les gisements ayant livré des fléchettes se situent dans le Grand SudOuest, essentiellement au nord de l'Aquitaine:

- en Gironde : à Pair-non-Pair (Daleau 1881 ; Cheynier et Daleau 1963) ;
- en Charentes : aux Vachons, abri II, couche 3 (Bouyssonie 1948, Bouyssonie et Sonneville-Bordes 1956) ;

- en Corrèze : à Noailles (Bardon et al. 1904), à La FontRobert (Bardon et al. 1906 ; Bardon et al. 1908) et à Puy Jarrige (Mazière 1980 ; Mazière et al.1984),

- en Dordogne : à Durand-Ruel (Pittard et Montandon 1912 ; Daniel et Schmider 1972), à l'Abri du Poisson, couche supérieure (Peyrony 1932), à La Gravette (Lacorre 1933, 1960), à la Roque Saint-Christophe ${ }^{2}$ (SonnevilleBordes 1960), à Laussel (Lalanne et Bouyssonie J. 1946), à l'Abri Vignaud (Sonneville-Bordes 1960), à l'Abri Pataud, couche 5 (Bricker 1995) et probablement à Oreille-d'Enfer (Pradel 1959),

- en Agenais : au Roc de Gavaudun, couche 2 (Monméjean et al. 1964) ${ }^{3}$.

Hors de cette zone géographique principale, seulement trois gisements ont livré des fléchettes. Deux occurrences languedociennes, la petite grotte de Bize et la Crouzade (Sacchi 1986), indiquent qu'il ne s'agit pas d'un phénomène uniquement aquitain et qu'il faille chercher d'autres facteurs à même d'expliquer la répartition des assemblages à fléchettes. Signalons enfin un dernier point, La Vigne Brun (Larue et al. 1955), jalon oriental de cette carte (fig. 1).

La première description fût donnée par les abbés Bardon et Bouyssonie à partir du matériel de la Grotte de Noailles : " lame très mince, de $57 \mathrm{~mm}$ de long, rendue pointue par retouches latérales, et qui devait être une pointe de flèche » (Bardon et al. 1904 p. 288). Ensuite à Durand-Ruel, ces pièces furent décrites comme de "petites lames minces dont le contour épouse assez fidèlement la forme d'une feuille ovalaire ou lancéolée » (Pittard et Montandon 1912 p. 31). F. Lacorre en donnera la première description complète (Lacorre 1933a), précisée par H. Delporte (Delporte 1972).

L'essentiel des découvertes eut lieu entre les années 1900 et 1940 (tabl. 1). Ce contexte de fouilles a suscité certaines remises en question, concernant l'intégrité des niveaux observés par D. de Sonneville-Bordes (1958) à La Gravette et à Vignaud, ainsi que par H. Delporte (Delporte 1972, 1976) à l'abri du Poisson, Laussel et la Roque Saint-Christophe (Rigaud 1988).Le cadre chrono-stratigraphique de la majorité des assemblages à fléchettes demeure donc mal défini. Parmi les sites stratigraphiquement fiables, citons l'Abri Pataud, Puy Jarrige et La Vigne Brun, derniers sites fouillés.

\section{Les datations}

Le manque de données concernant la position chronostratigraphique des fléchettes ne peut être compensé par un nombre suffisant de datations absolues. En effet, seuls deux gisements ont fait l'objet de mesures radiométriques, l'Abri

(2) Les fléchettes de la Roque Saint-Christophe ne proviennent pas des fouilles D. Peyrony (Peyrony 1939) mais des fouilles P. Fitte (Sonneville-Bordes 1960).

(3) Au Roc de Cavart des pièces sublosangiques sont interprétées comme des fléchettes (Le Tensorer 1981), cependant elles diffèrent quelque peu de la norme et ne seront donc pas retenues. 


\begin{tabular}{|ccc|}
\hline Gisements & Fouilleurs & Périodes \\
\hline \multirow{2}{*}{ Bize } & E. Genson & 1927 à 1929 \\
& Th. et Ph. Héléna & 1930 à 1933 \\
Crouzade & Th. Héléna et E. Genson & 1906 à 1931 \\
& Ph. Héléna & 1946 \\
Durand-Ruel & E. Pittard & 1906 à 1912 \\
& R. Daniel & 1919 \\
Font-Robert & Pierre, Comte et Comtesse de & 1905 \\
\hline Gravette & Thévenard & 1931 à 1954 \\
\hline Laussel & F. Lacorre & 1908 à 1914 \\
\hline Noailles & Dr Lalanne & 1903 \\
\hline Oreille-d'Enfer & L. Bardon, A. et J. Bouyssonie & 1941 \\
\hline Pair-non-Pair & L. Pradel & 1881 à 1889 \\
\hline Pataud & F. Daleau & 1958 à 1964 \\
\hline Poisson & H. L. Movius & 1917 \\
\hline Puy Jarrige & D. Peyrony & 1980 \\
\hline Roc de Gavaudun & G. Mazière & 1931 à 1937 \\
Roque Saint-Christophe & E. Monméjean & 1912 à 1913 \\
\hline Vachons & D. Peyrony & antérieures à 1900 \\
\hline Vignaud & J. Coiffard, & 1929 à 1937 \\
Vigne Brun & J. Bouyssonie & 1941 \\
\hline & D. Peyrony & 1977 à 1983 \\
\hline
\end{tabular}

Tableau 1 - Périodes de fouille des gisements ayant livré des fléchettes.

Table 1 - Excavation period in which flechettes have been found.

Pataud et La Vigne Brun. Pour le premier, la date retenue pour la couche 5, Front Lower 2, est la plus ancienne obtenue avec $28400 \pm 1100$ BP, OXA 169 (Bricker 1995). Cependant, les nombreuses datations effectuées situent l'occupation de ce niveau dans une fourchette chronologique beaucoup plus large entre $27545 \pm 320 \mathrm{BP}(\mathrm{Gx} 1370)$, $26720 \pm 460 \mathrm{BP}$ (Gx 1369), $25815 \pm 330 \mathrm{BP}(\mathrm{Gx} 1371)$ et $23600 \pm 800$ BP (W 151) (Bricker 1995). En ce qui concerne La Vigne Brun, les datations s'échelonnent entre 24900 \pm 2000 BP (Ly 391), $24900 \pm 900$ BP, $23500 \pm 1000$ BP (Ly 2640), $23450 \pm 690 \mathrm{BP}$ (Ly 2637), $23230 \pm 760 \mathrm{BP}$ (Ly 2639) et $21580 \pm 600$ BP (Ly 2638) (Combier 1980 ; Combier 1989 ; Evin 1982). Pour ce gisement, un effet de rajeunissement important des datations est à envisager, probablement lié à la mauvaise conservation des éléments osseux en contexte granitique. Les datations de ces deux sites s'avèrent donc beaucoup trop hétérogènes pour définir un cadre chronologique cohérent.

La position stratigraphique des niveaux à fléchettes en base de séquence à La Gravette, à l'Abri Pataud ainsi qu'à Willendorf (Felgenhauer 1959 ; Otte 1981) en Autriche, constitue donc toujours le meilleur argument en faveur de l'ancienneté de ce faciès.

\section{Les associations avec d'autres « fossiles directeurs »}

Si ce dernier point est acquis depuis longtemps (Peyrony 1933), la période de disparition des fléchettes dans les assemblages suscite en revanche certaines incertitudes, notamment liées à l'association de fléchettes avec les «fossiles directeurs » du Périgordien V.

\section{Les pointes de La Font-Robert}

Les « fossiles directeurs » des Périgordien IV et Va, fléchettes et pointes de La Font-Robert, furent retrouvés à plusieurs reprises dans un même ensemble archéologique, comme à Durand-Ruel (Daniel et Schmider 1972), La FontRobert (Bardon et al. 1908), Laussel (Lalanne et Bouyssonie J. 1946), les Vachons (abri II, couche 3) (Bouyssonie 1948 ; Bouyssonie et Sonneville-Bordes 1956), La Vigne Brun (unités OP10, O16, KL19 et secteur I) et dans le Jura Souabe, à Geissenklösterle (couche la) (Otte 1981). 
Ces associations au sein de mêmes ensembles stratigraphiques ne permettent pas d'apporter de réponse à une question pourtant névralgique dans la compréhension de l'évolution du Gravettien : quels rapports existent entre faciès à fléchettes et faciès à pointes de La Font-Robert ?

Le site du Jura Souabe présente la seule succession stratigraphique bien documentée de ces deux outils. La couche la renferme deux fléchettes et une pointe de La Font-Robert, la couche lb, sous-jacente, une seule fléchette. Cependant, les études ultérieures remirent en question les subdivisions distinguées à la fouille (Hahn et al. 1985).

Dans le gisement girondin de Pairnon-Pair, les fléchettes se rencontrent dans les couches $3,4, C-D, D, K$, sous le niveau comprenant l'unique pointe de La Font-Robert, couche 7 . Cette occurrence pourrait être un des rares arguments stratigraphiques directs en faveur de l'antériorité des fléchettes sur les pointes de La Font-Robert, si les données stratigraphiques permettaient de définir précisément la position respective de ces objets (Malvesin 1948 ; Lenoir 1983).

Pour La Vigne Brun, la situation est différente. Si un seul ensemble archéologique a été mis en évidence lors de la fouille, de nombreuses indications comme l'existence de couches d'ocre, d'épandages charbonneux et de pavages d'ossements, orientaient la perception du remplissage comme une succession de dépôts archéologiques sur plus de $50 \mathrm{~cm}$ d'épaisseur (Combier 1980).

L'analyse spatiale réalisée par E. Nonet permît de vérifier ces observations et de reconnaître plusieurs stades dans le fonctionnement d'une structure, l'unité OP10 (Nonet 2004). L'existence d'une microstratigraphie pourra alors éventuellement permettre de définir la position stratigraphique relative de ces deux " fossiles directeurs ».

\section{Les burins de Noailles}

Les fléchettes furent également retrouvées avec des burins de Noailles dans plusieurs gisements dont le Poisson, Oreille d'Enfer (Pradel 1959), Durand-Ruel (Daniel et Schmider 1972) et le Roc de Gavaudun, couche 2 (Monméjean et al. 1964). À la Roque Saint-Christophe, des burins du Raysse viennent s'ajouter, amenant J.-P. Rigaud (Rigaud 1988) à rajeunir cet ensemble attribué à un Périgordien IV (Sonneville-Bordes 1960).

\section{Conclusion}

À l'aune de ces fouilles, les premiers stades du Gravettien ne firent pas l'objet d'une approche globale visant à définir la nature de ces assemblages ni les liens existants entre eux. Seules les principales collections de fléchettes firent l'objet d'études typo-métriques ciblées révélant au-delà de l'existence d'une norme commune, les particularités des fléchettes pour La Gravette (Lacorre 1960 ; Delporte 1972), l'Abri Pataud (Bricker 1973, 1974 ; Perpère 1992) et en Autriche, Aggsbach (Otte 1981).
Dans la compréhension de l'évolution des phases anciennes du Gravettien, un problème demeure donc : l'absence de stratigraphie complète. Cette lacune, contournée par D. Peyrony en superposant la stratigraphie de la Ferrassie à celle de La Gravette (Peyrony 1933), constitue toujours un écueil. Les modèles récents de structuration du Gravettien en rendent compte (Bosselin et Djindjian 1994) sans parvenir à apporter de réponse quant à la nature des liens existant entre faciès à fléchettes, à pointes de La Font-Robert et à gravettes seules. Cette évolution qui ne semble pas enregistrée dans la stratigraphie de l'Abri Pataud, peut donc trouver des éléments de compréhension dans les gisements de Geienklösterle et de La Vigne Brun.

Toutefois, si une perduration des fléchettes jusqu'aux éléments à pointes de La Font-Robert reste envisageable, l'association des fléchettes avec des burins de Noailles pourrait être liée à l'ancienneté des fouilles et au manque inhérent de données stratigraphiques, ce qui ne permet pas de statuer avec fiabilité sur le sens de ces associations dont les origines peuvent être multiples. La présence épisodique de fléchettes au sein d'assemblages mal caractérisés stratigraphiquement et culturellement ne doit pas masquer pour autant sa forte valeur diagnostique d'un point de vue chronologique.

\section{Nouvelles perspectives}

Compte tenu de l'indigence des données existantes sur les premières phases du Gravettien, un retour aux collections de référence afin d'appréhender cet épisode du Paléolithique supérieur sur des bases nouvelles s'impose donc. Le choix a porté sur deux gisements : le niveau bayacien de La Gravette, puis La Vigne Brun. L'objectif est d'évaluer dans quels systèmes techniques s'insère la production de fléchettes dans des contextes où la fléchette constitue la seule armature, puis lorsqu'elle est associée à d'autres «fossiles directeurs » du Gravettien. La question de l'existence du Bayacien pourra ainsi être posée.

\section{La Gravette}

L'importante monographie consacrée à ce gisement deux fois éponyme révèle la richesse et l'intérêt de cette collection (Lacorre 1960). Le niveau bayacien se distingue nettement des trois niveaux "gravétiens» sus-jacents par la réalisation d'objectifs techniques forts différents.

Bien que la collection constituée par F. Lacorre comporte certains biais étant donné l'ancienneté des fouilles, la quasi-absence de fraction fine et la faible représentation des produits bruts, la constitution actuelle de l'assemblage permet tout de même de restituer avec une certaine acuité la nature des procédés techniques mobilisés par les tous premiers artisans gravettiens (fig. 2).

\section{Description}

La production essentiellement tournée vers l'obtention de fléchettes confère un aspect extrêmement léger à cette industrie. La proportion d'éléments appartenant à 

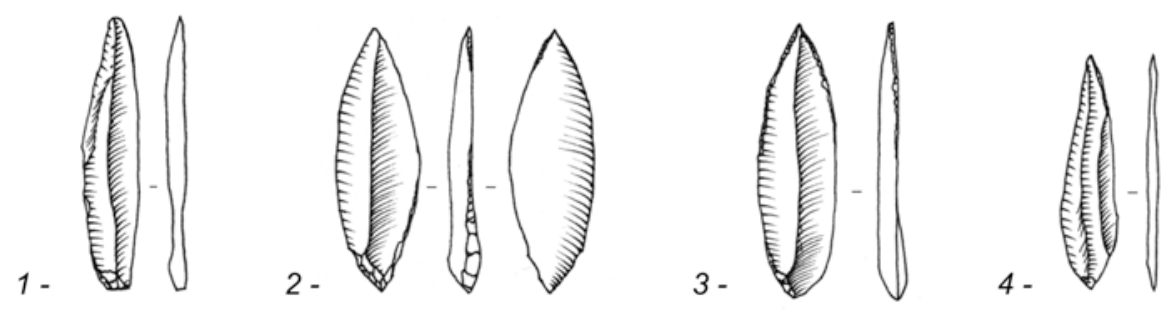

Figure 2 -

Fléchettes,

niveau bayacien

de La Gravette.

Figure 2 -

Fléchettes,

bayacian level

of La Gravette.

5 -

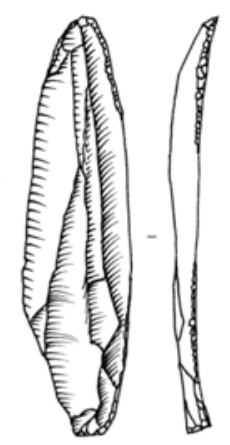

$6-$
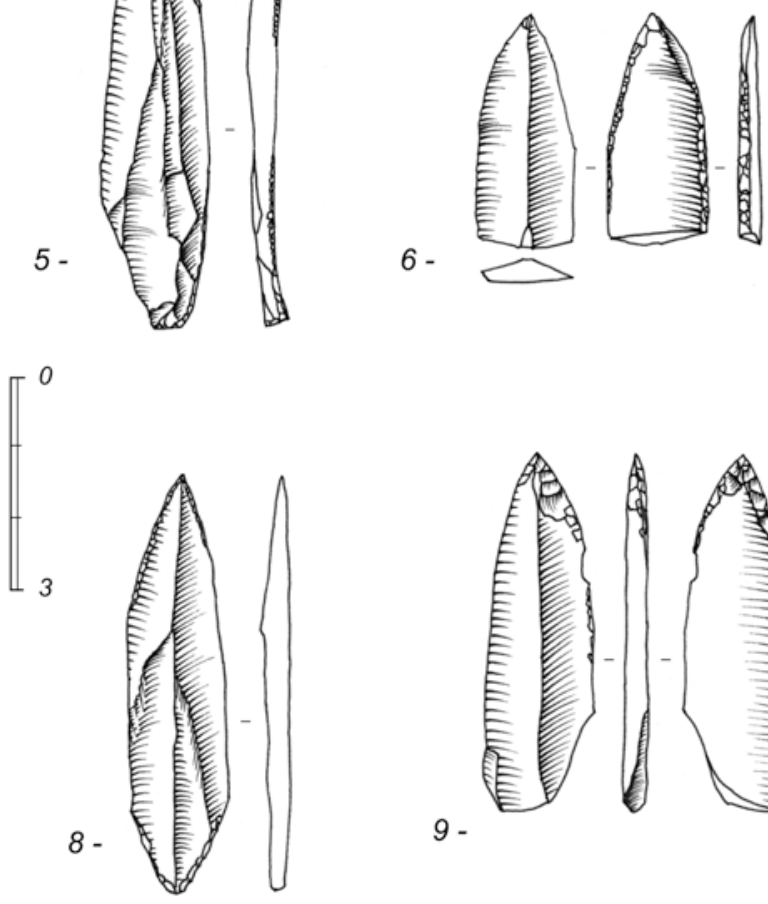

9 -

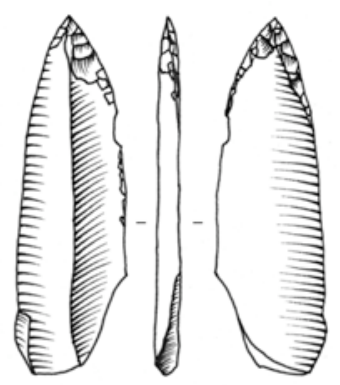

ce système technique est prédominante, puisqu'elle rassemble $77 \%$ de l'assemblage. La série comprend 145 fléchettes dont 117 sont entières. Les pièces subentières sont peu nombreuses avec 19 éléments dont il manque l'extrémité distale et 4 , la base. Enfin, 4 fragments proximaux sont présents pour un seul distal.

La dimension des fléchettes révèle un degré de dispersion variable selon le paramètre considéré. La longueur constitue le principal élément de variation, se répartissant entre 26 et $73 \mathrm{~mm}$ de long, pour une moyenne de $48,61 \mathrm{~mm}$ (fig. 2.). Les largeurs des fléchettes sont comprises entre 9 et 15 $\mathrm{mm}$, enfin l'épaisseur des produits témoigne d'une nette normalisation puisque $85 \%$ des supports mesurent entre 2 et $3 \mathrm{~mm}$ d'épaisseur. Henri Delporte a démontré la variabilité de l'aménagement des fléchettes (Delporte 1972). Si la retouche demeure semi-abrupte, sa localisation sur le support et la face investie varient de manière importante. L'auteur observe le caractère majoritairement direct de la retouche, qui investit fréquemment le bord droit.
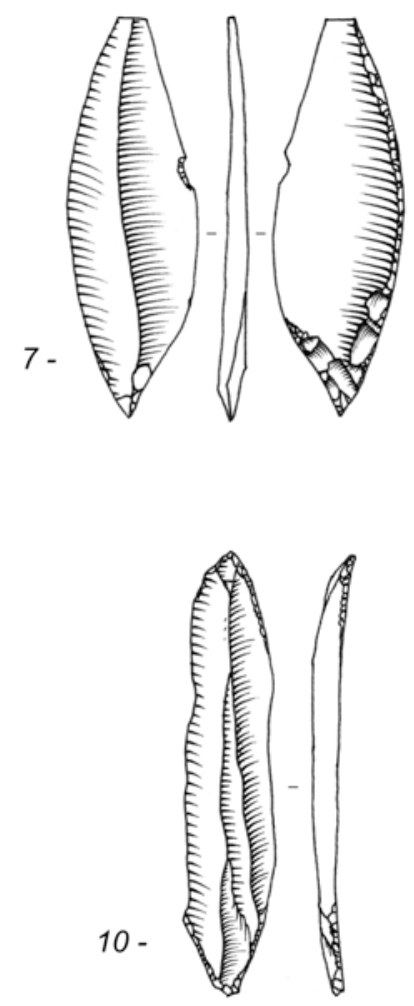

\section{Production}

La régularité des supports de fléchettes sous-entend l'application de modes opératoires très investis techniquement et une standardisation des procédés d'obtention.

Un schéma de production très normé apparaît destiné à l'obtention de supports rectilignes. Le débitage est mené à partir d'un plan de frappe principal, sur un volume entièrement mis en forme (fig. 3). Le dos est régularisé par une série d'enlèvements ; le plan de frappe, d'angulation ouverte, reste lisse. La base et les flancs sont aménagés à partir d'un plan de frappe opposé décalé. La table est rectiligne, large, très peu cintrée, se resserrant en partie distale car la convergence des enlèvements, associée à la présence de la corniche du plan de frappe opposé créée un dièdre qui canalise l'onde de choc. De ce fait, en partie proximale, depuis la zone de contact du percuteur (vraisemblablement tendre organique), l'onde de choc part en s'évasant, s'élargit en partie mésiale, puis se resserre en partie distale. Le support 

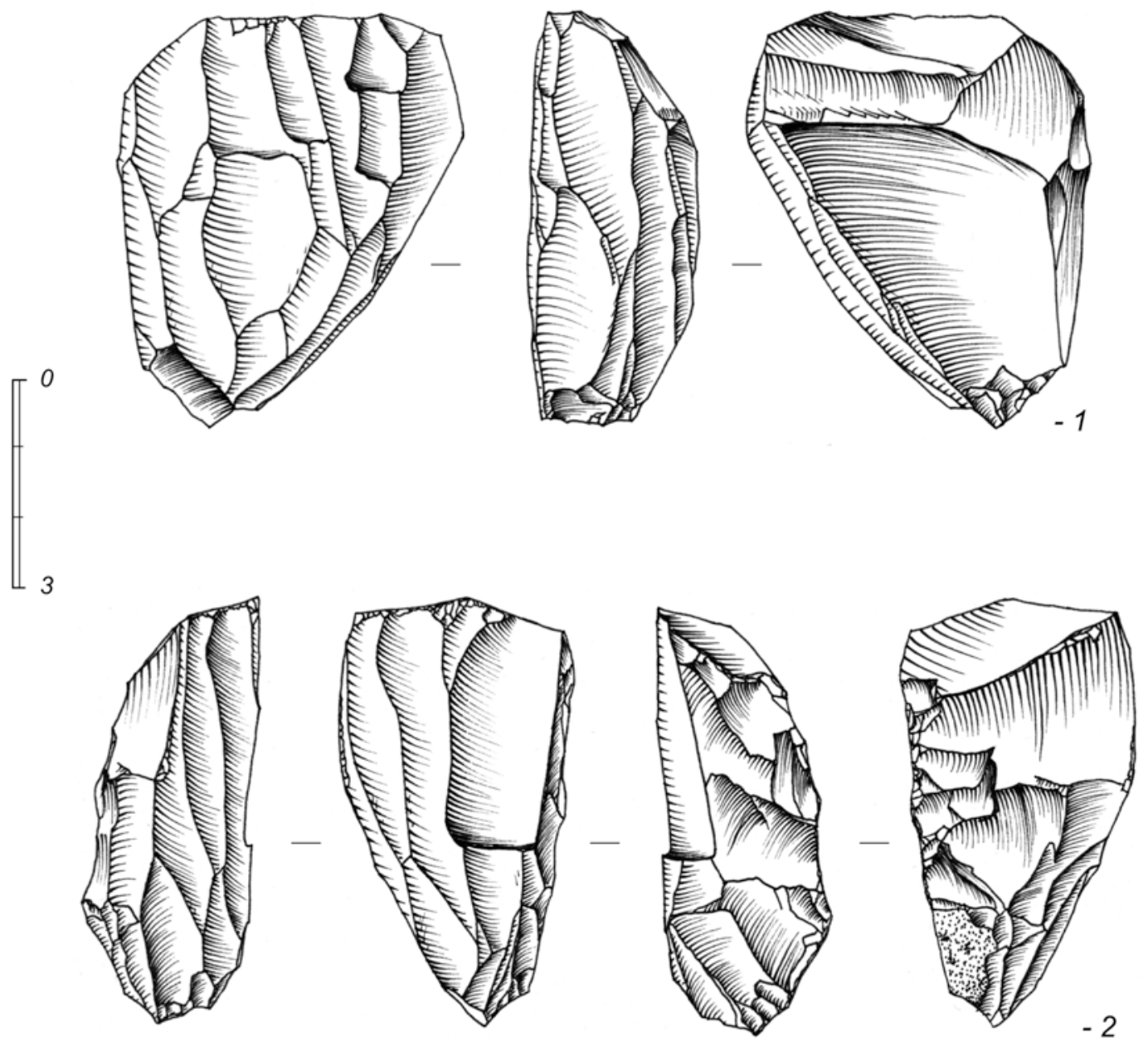

Figure 3 - Nucléus à fléchettes, niveau bayacien de La Gravette.

Figure 3 - Fléchettes cores, bayacian level of La Gravette.

ainsi obtenu possède donc de manière intrinsèque la morphologie sublosangique des fléchettes. Les produits ne sont donc pas des petites lames mais bien des fléchettes. Tout l'art de ce débitage réside donc dans la manière de faire varier en différents points l'étalement latéral de l'onde de choc. Cet aspect est également tributaire de la morphologie des flancs, volontairement définis de manière légèrement convexes, notamment par les enlèvements issus du plan de frappe opposé décalé.

À titre d'hypothèse, I'utilisation d'un plan de frappe opposé peut être mise en relation avec une volonté de ne pas délimiter la table à partir du plan de frappe principal. En effet, l'investissement des flancs à partir du plan de frappe principal induirait une torsion des supports situés en bord de table. Or, les supports latéraux sont utilisés pour la confection de fléchettes et aucune d'entre elles ne présente de torsion en partie proximale. De fait, l'ensemble des supports extraits depuis le plan de frappe principal peut être considéré comme des produits.
Dans les premiers stades, les produits obtenus sont relativement allongés mais tendent à s'élargir avec la réduction du volume et l'aplatissement de la table. Ces supports présentent une ou plusieurs nervures, pas nécessairement centrées, ni parallèles et des bords convexes. Le produit recherché possède toutes les caractéristiques de l'outil, de morphologie sublosangique, peu épais, large par rapport à sa longueur, à extrémité distale aigue.

En marge de cette production majoritaire, certains supports se dissocient de cette norme. Ces produits correspondent véritablement à de petites lames rectilignes, sveltes, élancées, à bords parallèles (fig. 4). Ces supports proviennent de nucléus dont les caractéristiques architecturales s'avèrent foncièrement différentes. La table de ces nucléus est beaucoup plus longue, très étroite et délimitée latéralement par des flancs abrupts, lisses et parallèles. Le volume est géré à partir de deux plans de frappes, opposés dans ce cas. D'après l'investissement de la table et la chronologie des enlèvements, il apparaît que ces deux 

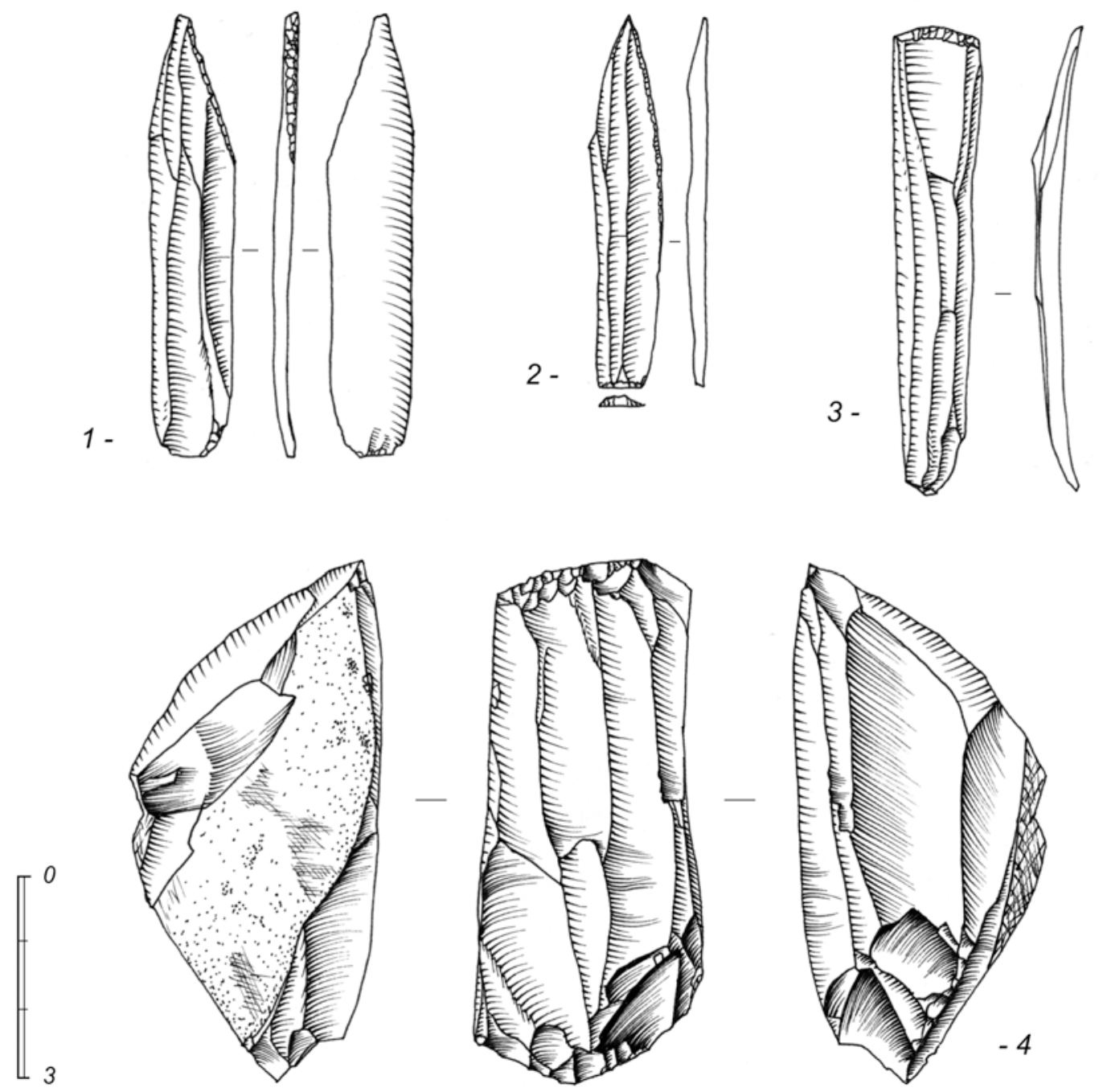

Figure 4 - Produits et nucléus correspondant à la chaîne opératoire de petites lames, niveau bayacien de La Gravette.

Figure 4 - Tools and core resulting from the small blades «chaine opératoire», bayacian level of La Gravette.

plans de frappe ne partagent pas les mêmes objectifs. Une alternance est identifiable entre l'extraction de supports longs et rectilignes et des supports plus courts et moins réguliers (fig. $4 n^{\circ}$ 5). Ces deux plans de frappe sont donc hiérarchisés, l'un servant à la production stricto sensu, l'autre à l'entretien du volume, de la carène.

Les produits obtenus dans ce cas sont de réelles lames légères à bords parallèles, acuminées par la transformation. Observons que les deux fléchettes de la série portant des négatifs d'enlèvements opposés correspondent à ces supports (fig $4, n^{\circ} 2$ ). Leur faible représentation masque le statut économique de cette production dans l'assemblage. Certaines de ces petites lames sont transformées en grattoirs, ce qui n'est pas le cas pour les supports extraits selon le schéma précédent. Ces deux modes de production présentent donc des divergences fortes portant sur la nature des procédés techniques mobilisés, la morphologie des produits et leur vocation fonctionnelle.

\section{Conclusion}

Ces premiers éléments de lecture des modes de production de fléchettes rendent compte d'un investissement en soin important dans le débitage par la structuration du volume, la mise en place de plans de frappe à fonctions très différenciées, la régularité des supports, ce que traduit l'existence de schémas opératoires complexes et spécialisés. L'obtention de fléchettes constitue l'objectif principal de la production. La seule composante autre correspond à une production laminaire d'appoint, plus robuste, sans lien technique ni fonctionnel avec les schémas présentés, destinée à alimenter l'outillage de fonds commun.

Aucun élément ne se rapporte donc à une production de pièces à dos. De plus, les modalités d'obtention des pointes de La Gravette des couches supérieures s'avèrent forts différentes. Le niveau bayacien de La Gravette apparaît donc comme un assemblage techniquement homogène. 
L'absence de fraction brute et la sous-représentation des fragments de fléchettes induisent un certain biais dans la compréhension de l'occupation mais ne remettent pas en question l'intégrité de cet assemblage dans sa composition majoritairement orientée vers la production de fléchettes à l'exclusion de tout autre type d'armature.

\section{La Vigne Brun}

Le gisement de La Vigne Brun a livré une importante occupation gravettienne. Fouillé par J. Combier, sur $450 \mathrm{~m}^{2}$, l'espace s'organise sous la forme de structures circulaires creusées dans le substrat et délimitées par un bourrelet de terre issue du creusement et de nombreux blocs de granit de grandes dimensions (Combier 1980). L'industrie lithique, abondante, est réalisée sur des roches d'origines très lointaines, provenant d'un espace géographique correspondant au pourtour septentrional du Massif central, mais également sur des matériaux plus locaux, issus des formations jurassiques du Bassin de la Loire, en aval du site (Masson 1981). Les nucléus ayant transité sur le site proviennent des formations turoniennes inférieures du Cher, crétacées et oligocènes du sud du Bassin parisien ainsi que des argiles à silex du Mâconnais.

\section{Description}

Les études concernant les unités KL19 (Digan 2001) et OP10 (Nonet 2002 ; Pesesse 2003) mettent en évidence la place centrale occupée par les pointes de La Gravette dans la structuration du système technique. La production de cette armature in situ influe sur le choix et le mode d'introduction des matériaux sur le gisement, et conditionne la mise en place de procédés techniques élaborés, visant à obtenir des lames et des lamelles rectilignes, élancées, pointues, à bords vifs et réguliers. Dans ces unités, la proportion de pointes de La Gravette représente respectivement $34 \%$ et $51 \%$ de l'outillage. Cette prédominance des pointes de La Gravette n'exclut pas pour autant la présence d'autres types d'armatures. Dans le cas de l'unité OP10, la population de fléchettes représente 12 éléments pour 871 outils, dont 448 pointes de La Gravette, 78 pointes à dos alternes et quatre pointes de La Font-Robert (Pesesse 2003). À l'échelle du gisement, cette très faible représentation des fléchettes s'observe également : 32 pièces sont pour l'instant décomptées pour un total avoisinant plusieurs dizaines de milliers de pièces. Les fléchettes mesurent entre 25 et $75 \mathrm{~mm}$ de long, 9 et $16 \mathrm{~mm}$ de large et 1,5 et $6 \mathrm{~mm}$ d'épaisseur (fig. 5).

\section{Production}

Les supports transformés se caractérisent par leur rectitude, par une section fréquemment symétrique, par une certaine régularité. Ils correspondent aux produits de première intention, extraits au centre d'une table régulière pour la majorité d'entre eux. Néanmoins, une sélection de supports beaucoup moins réguliers apparaît également, produits de seconde intention ou véritables sousproduits du débitage laminaire (lames décentrées, souscrêtes antérieures).
Les caractéristiques morphologiques, métriques et pétrographiques des supports ne permettent pas de dissocier technologiquement les productions de pointes de La Gravette et de fléchettes. Ces éléments vont en faveur d'une transformation différenciée des produits issus d'un même schéma opératoire en fonction de leurs caractéristiques propres dans la mesure où l'utilisation de la percussion induit une source de variabilité relative au sein d'une production, même normée.

Ainsi, l'extraction d'un dièdre, d'une nervure saillante confère au support une morphologie triangulaire, une section symétrique, ce qui incarne bien l'image de la fléchette. Cet aspect est également lié à la convergence distale des enlèvements. De plus, bien que la production de supports d'armatures rectilignes soit conceptuellement très normée, les Gravettiens font preuve d'une certaine souplesse en mobilisant un panel d'options techniques, à valeur de modalités, liées notamment à la morphologie initiale des blocs, influant sur la délimitation plus ou moins abrupte de la table, et subséquemment sur l'investissement des flancs. Ces variations sur le thème entraînent donc une source supplémentaire potentielle de différenciation des produits. Ces supports proviennent majoritairement d'une chaîne opératoire unipolaire convergente. Certains éléments documentent l'utilisation ponctuelle d'un plan de frappe opposé dans un cadre d'entretien (Pesesse 2003).

De rares fléchettes présentent une morphologie assez particulière par leur équilibre, leur symétrie, leur finesse et leur forme presque losangique (fig. $5, n^{\circ} 8$ ), permettant d'envisager éventuellement une production dissociée. Cet aspect, par le faible nombre de pièces et la quasi-absence de nucléus en matières allochtones compte tenu du statut du gisement, demeure hypothétique.

La production s'opère donc dans un continuum technique et fonctionnel entre débitages laminaires dont les supports sont destinés à l'outillage de fonds commun, puis débitages de petites lames et de lamelles transformées en armatures.

\section{Conclusion}

Au sein de l'équipement lié aux activités d'acquisition des chasseurs de La Vigne Brun, la fléchette occupe donc une place marginale par le faible nombre d'éléments présents et par le fait que les produits sélectionnés proviennent de schémas opératoires en priorité destinés à fournir des supports de pointes de La Gravette. La situation s'avère donc très différente de La Gravette où la fléchette structure la production lithique. Dans ces gisements, ces armatures sublosangiques relèvent de deux normes de productions très différentes, par la nature et l'imbrication des chaînes opératoires à finalités cynégétiques et domestiques, dissociées à La Gravette et intégrées à La Vigne Brun. Pourtant la transformation des supports s'opère selon les mêmes conventions techniques dans la réalisation d'une armature axiale à retouche semi-abrupte, limitée aux parties basales et 

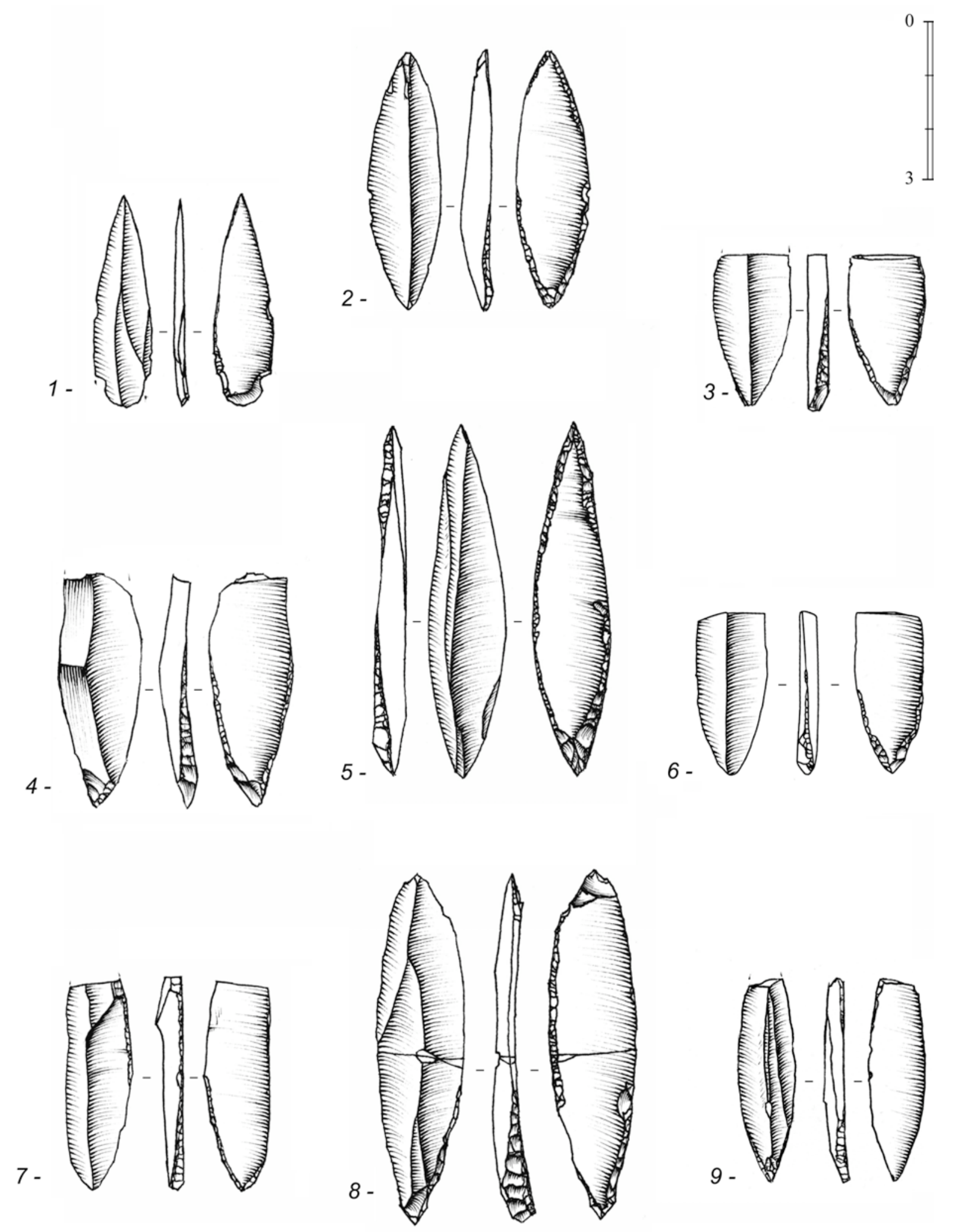

Figure 5 - Fléchettes, La Vigne Brun.

Figure 5 - Fléchettes, La Vigne Brun. 
apicales. À La Vigne Brun, cette retouche confère aux supports la morphologie sublosangique de la fléchette, caractéristique définie lors du débitage à La Gravette

De La Gravette à La Vigne Brun, durant cet intervalle de temps, les systèmes techniques lithiques gravettiens furent complètement restructurés autour d'une nouvelle arme de chasse, la pointe de La Gravette. Absente dans le Bayacien de La Gravette, elle constitue l'essentiel de l'équipement cynégétique des chasseurs de chevaux de La Vigne Brun. Toute la production d'armatures se trouve modifiée techniquement et économiquement. Seule cette modalité de transformation de l'armature présente une certaine pérennité. Face à la diversité des armatures de La Vigne Brun pointes de La Gravettes, microgravettes, nanogravettes, pointes à dos alternes et pointes de La Font-Robert - la fléchette peut éventuellement apparaître comme un complément fonctionnel, lié à son caractère d'armature axiale.

Peut-être faut-il chercher plus loin les raisons de la perduration du concept de fléchette dans les sociétés gravettiennes postérieures au Bayacien. Elle peut difficilement s'appréhender comme une survivance anecdotique si l'on s'accorde sur le statut des armes de chasse en matière minérale dans certaines sociétés paléolithiques, gravettiennes et solutréennes notamment. La fléchette peut alors apparaître comme un marqueur identitaire fort des premiers gravettiens et, à ce titre, avoir perduré quelque peu dans la société gravettienne alors même que l'ensemble des normes techniques avait évolué.

Le Bayacien de La Gravette révèle une homogénéité du point de vue de la corrélation entre les processus techniques et les objectifs, et la complémentarité fonctionnelle entre les trois schémas opératoires définis. Cet assemblage présente donc des caractéristiques originales par la nature et la structuration des procédés techniques mobilisés par les premiers gravettiens. Dans cette acception, aucun autre équivalent n'est encore reconnu. Le particularisme de cette industrie, doublé d'une position chrono-stratigraphique charnière mérite donc son individualisation sous le terme de Bayacien proposé par F. Lacorre. En ce sens, seul le niveau de La Gravette se rapporte au Bayacien. La présence de quelques fléchettes, comme à Vigne Brun ou à Puy Jarrige, lorsqu'elle n'est pas le fruit de mélanges, n'est pas suffisante pour parler de Bayacien. Toutefois, si la perduration de ces armatures peut être le signe du maintien des traditions, les processus d'évolution technique entre le Bayacien et le Gravettien ancien à fléchettes demeurent à définir.

\section{BIBLIOGRAPHIE}

BARDON L., BOUYSSONIE J., BOUYSSONIE A. 1904 Monographie de la grotte de Noailles (Corrèze). Revue de l'école d'Anthropologie de Paris, t 14, p. 283-294.

BARDON L., BOUYSSONIE A. et J. 1906 - La Grotte de La Font-Robert près Brive (Corrèze), Congrès International d'Anthropologie et d'Archéologie Préhistorique, Compte Rendu de la $13^{\circ}$ session, Monaco, t. 2, p. 172-184.
BARDON L., BOUYSSONIE A. et J. 1908 - Station préhistorique du château de Bassaler. La Grotte de La FontRobert, Bulletin de la Société Scientifique, Historique et Archéologique de la Corrèze, t. 30, p. 315-331.

BOSSELIN B. 1996 - Contribution de l'abri Pataud à la chronologie du Gravettien français, Bulletin de la Société Préhistorique Française, tome 32, n² 2, p. 183-194.

BOSSELIN B. et DJINDJIAN F. 1994 - La chronologie du Gravettien français, Préhistoire Européenne, vol 6, p. 77-115.

BOUYSSONIE J. 1948 - Un gisement aurignacien et périgordien, les Vachons (Charente), L'Anthropologie, tome 52, p. 1-42.

BOUYSSONIE J. et SONNEVILLE-BORDES D. 1956 - L'abri $\mathrm{n}^{\circ} 2$ des Vachons, gisement aurignacien et périgordien, commune de Voulgézac (Charente), Congrès Préhistorique de France, $\mathrm{XV}^{\circ}$ session, Poitiers-Angouléme, p. 271-309.

BRICKER H. M. 1973 - The Perigordian IV and related cultures in France, Harvard University, Department of Anthropology, Ph. D. Dissertation, 1849 p.

BRICKER H. M. 1974 - La contribution de l'Abri Pataud à la question bayacienne, Congrès Préhistorique de France, $X X^{\circ}$ Session, Provence, p. 48-52.

BRICKER H. M. (dir.) 1995 - Le Paléolithique supérieur de l'Abri Pataud (Dordogne) : les fouilles de H. L. Movius, Paris, Maison des Sciences de l'Homme, Documents d'Archéologie Française, $n^{\circ}$ 50, 328 p.

CHEYNIER A. et DALEAU F. 1963 - La caverne de Pair-nonPair, (Gironde), Documents d'Aquitaine, Publication de la Société archéologique de Bordeaux, Bordeaux, 220 p.

COMBIER J. 1980 - Circonscription Rhône-Alpes, Gallia Préhistoire, vol. 23, p. 511-515.

COMBIER J. 1989 - Aurignacien et Périgordien dans l'Est de la France, In Le Temps de la Préhistoire, sous la direction de J.-P. Mohen, XXIII Congrès Préhistorique de France, tome 1, p. 274-275.

DALEAU F. 1881 - La grotte de Pair-non-Pair, Association Française pour l'Avancement des Sciences, Alger, $10^{\circ}$ Session, Alger, p. 755.

DANIEL R. et SCHMIDER B. 1972 - L'abri Durand-Ruel, près Brantôme (Dordogne) et ses problèmes stratigraphiques, Gallia Préhistoire, tome 15, p. 323-337.

DELPORTE H. 1972 - L'Aurignacien et le Bayacien de La Gravette : Mise en oeuvre statistique et problèmes posés, Bulletin de la Société Préhistorique Française, tome 69, Etudes et travaux, fascicule 1, p. 337-346.

DELPORTE H. 1976 - L'organisation du Périgordien supérieur en France, et ses rapports avec le Périgordien d'Europe occidentale, ERAUL n 13, Liége, fascicule I, p. 83 à 106. 
DIGAN M. 2001 - Le gisement gravettien de La Vigne-Brun (Loire) : première étude de l'industrie lithique de KL19, Mémoire de doctorat, Lille I, 2 vol.

DJINDJIAN F. et BOSSELIN B. 1994 - Périgordien et Gravettien : l'épilogue d'une contradiction ?, Préhistoire Européenne, vol 6, p. 117-131.

EVIN J. 1982 - Datations par radiocarbone du site de La Vigne Brun à Villerest (Loire), In : Les habitats du Paléolithique supérieur : Actes du colloque international en hommage au professeur André Leroi-Gourhan, Roanne-Villerest, 22-24 juin 1982, J. Combier, Lyon, 2 vol, p. 148-149.

FELGENHAUER F. 1956-1959 - Willendorf in der Wachau, Monograhie der Paläolith-Fundstellen I-VII. Mitteilungen der Prähistorischen Kommission der österreichischen Akademie der Wissenschaft, VIII und IX Band, 204 p.

HAHN J. et OWEN L. R. 1985 - Blade technology in the Aurignacian and Gravettian of Geienklösterle Cave, Southwest Germany, World Archeology 17, p. 61 - 75.

KLARIC L. 2003 - L'unité technique des industries à burins du Raysse dans leur contexte diachronique. Réflexions sur la diversité culturelle au Gravettien à partir des données de la Picardie, d'Arcy-sur-Cure, de Brassempouy et du Cirque de la Patrie, Paris, Université de Paris I - Panthéon Sorbonne, Thèse de doctorat, $426 \mathrm{p}$.

LACORRE F. 1933a - Les armatures de flèches de La Gravette, $\mathrm{XV}^{\circ}$ Congrès International Anthropologie et d'Archéologie Préhistorique de Paris, 20 à 27 septembre 1931, p. 409-415.

LACORRE F. 1933b - Les armatures de flèches de La Gravette. Niveau et industries auxquelles elles se rattachent. Procès-verbaux de la Société linéenne de Bordeaux, séance du 21 juin 1933, p. 78-87.

LACORRE F. 1959 - Tableau chronologique du Périgordien et de l'Aurignacien (suite) Classification des industries à éclats et lames à bord abattu, Bulletin de la Société Préhistorique Française, t 55, p. 433 - 440.

LACORRE F. 1960 - La Gravette, le Gravétien et le Bayacien, Laval, Imprimerie Barneoud.

LALANNE J. G. et BOUYSSONIE J. 1941 à 1946 - Le gisement paléolithique de Laussel. Fouilles du Docteur Lalanne, L'Anthropologie, tome 50, p. 1-163.

LARUE M., COMBIER J. et ROCHE J. 1955 - Les gisements périgordien et magdalénien du Saut-du-Perron (Loire), L'Anthropologie, tome 59, p.401-428.

LENOIR M. 1983 - Le Paléolithique des basses vallées de la Dordogne et de la Garonne, Thèse de Doctorat, Bordeaux I.
LE TENSORER J.-M. 1981 - Le Paléolithique de l'Agenais, Cahiers du Quaternaire $n^{\circ} 3$, Editions du CNRS.

MALVESIN-FABRE G. 1948 - La stratigraphie de Pair-nonPair, Procés-Verbaux de la Société Linnéenne de Bordeaux.

MASSON A. 1981 - Pétroarchéologie des roches siliceuses. Intérêt en Préhistoire. Thèse $3^{\circ}$ cycle, Université Lyon I, n¹035, 101 p., 32 fig., 7 pl.

MAZIERE G. 1980 - Circonscription du Limousin, Gallia Préhistoire, tome 23, fascicule 2, p. 362-365.

MAZIERE G., RAYNAL J.-P., DEMARS P.-Y. et COURTY M.-A. 1984 - Le gisement paléolithique supérieur de Puyjarrige 2 (Brive, Corrèze), Revue Archéologique du Centre de la France, t. 23, vol 1, p. 21-67.

MONMEJEAN E., BORDES F. et SONNEVILLE-BORDES D. (de) 1964 - Le Périgordien supérieur à burins de Noailles du Roc de Gavaudun (Lot-et-Garonne), L'Anthropologie, tome 68, p. 253 à 316.

NONET E. 2002 - Les objectifs des débitages laminolamellaires de l'unité OP10 de La Vigne Brun (Loire), Mémoire de Maîtrise, Université Aix-Marseille I.

NONET E. 2004 - Vision diachronique d'une unité d'habitation gravettienne : L'unité OP10 de La Vigne Brun (Loire, France), Mémoire de DEA, Université Aix-Marseille I, 182 p.

OTTE M. 1981 - Le Gravettien en Europe centrale, Dissertationes Archaeologicae Gandenses, vol XX, De Tempel, Brugge.

PERPERE M. 1992 - Contribution à l'étude des pointes de traits périgordiennes : les fléchettes, Préhistoire Européenne, vol 2, p. 89-95.

PESESSE D. 2003 - Approche du comportement technique au Gravettien : l'industrie lithique de l'unité OP10 de La Vigne Brun, mémoire de DEA, Université Aix-Marseille I.

PEYRONY D. 1932 - Les abris Lartet et du Poisson à Gorge d'Enfer (Dordogne), L'Anthropologie, tome 42, p. 241-268.

PEYRONY D. 1933 - Les industries aurignaciennes dans le bassin de la Vézère, Bulletin de la Société Préhistorique Française, tome 30, p. 543-559.

PEYRONY D. 1939 - Fouilles de la Roque SaintChristophe, Bulletin de la Société Historique et Archéologique en Périgord, t. 66, p. 218-269.

PITTARD E. et MONTANDON R. 1912 - Outillage microlithique de la station aurignacienne « Durand-Ruel » (vallon des Rébières), Dordogne, in Compte rendu de la XIV ${ }^{\circ}$ session du Congrès International d'Anthropologie et d’Archéologie Préhistoriques, Genève, p. 479-488. 
PRADEL L. 1959 - Le niveau de Noailles d'Oreille-d'Enfer, commune des Eyzies-de-Tayac (Dordogne), Bulletin de la Société Préhistorique Française, tome 55, p. 228-235.

RIGAUD J.-P. 1988 - The Gravettian Peopling of southwestern France, in Upper Pleistocene Prehistory of Western Eurasia, sous la direction de $\mathrm{H}$. Dibble et $\mathrm{A}$ Montet-White, University Museum Monograph 54, University of Pensylvania, Philadelphia, p. 386-396.
SACCHI D. 1986 - Le Paléolithique supérieur du Languedoc occidental et du Roussillon, XXI ${ }^{\circ}$ supplément à Gallia Préhistoire, Editions du CNRS.

SONNEVILLE-BORDES D. (de) 1958 - Problèmes généraux du Paléolithique supérieur dans le Sud-ouest de la France, L'Anthropologie, tome 62, p. 413-451.

SONNEVILLE-BORDES D. (de) 1960 - Le Paléolithique supérieur en Périgord, Bordeaux, Delmas, 2 vol., 558 p. 
